\title{
Antibiotic-loaded hydroxyapatite blocks in the treatment of experimental osteomyelitis in rats
}

\author{
M. ITOKAZU, T. OHNO, T. TANEMORI, E. WADA, N. KATO* and K. WATANABE* \\ Department of Orthopaedic Surgery and *Institute of Anaerobic Bacteriology, Gifu University School of \\ Medicine, 40-Tukasamachi, Gifu 500, Japan
}

\begin{abstract}
A novel drug delivery system for osteomyelitis was developed in which a porous hydroxyapatite block (HAB) is loaded with antibiotic by a centrifugation method. In this study, implantation of HABs loaded with the aminoglycoside antibiotic, arbekacin, were tested in established Staphylococcus aureus osteomyelitis in the proximal tibia of rats after debridement of the marrow cavity. The animals were observed for radiographic signs of infection and tissue was examined histologically. The infections were also evaluated by bone cultures. Bacterial counts were statistically lower in rats implanted with an antibiotic-loaded HAB than in those given a drug-free HAB. Radiographical and histological observations also showed beneficial effects of the antibiotic-loaded implant. The results suggest that the centrifugation method for loading HABs provides a simple drug delivery system. These antibiotic-loaded HABs may be useful for filling grafts in osteomyelitis.
\end{abstract}

\section{Introduction}

Despite various methods of therapy, chronic osteomyelitis still remains one of the most difficult diseases to treat. Hydroxyapatite $\left(\mathrm{Ca}_{10}\left(\mathrm{PO}_{4}\right)_{6}(\mathrm{OH})_{2} ;\right.$ mol.wt 1004.63) has excellent biocompatibility and a porous structure. It can be used to fill the dead space after curettage and provides a drug delivery vehicle for antibiotics. We have reported a novel drug-delivery system for osteomyelitis in which a hydroxyapatite block (HAB) is loaded with antibiotics by a centrifugation method, and then gives a slow release of antibiotic activity in vitro [1].

The present in-vivo study assessed the efficacy of antibiotic-loaded HABs in a rat model of osteomyelitis caused by Staphylococcus aureus. Efficacy was evaluated by residual bacterial counts after treatment, radiographs and histological changes in experimental osteomyelitis in the tibia of rats.

\section{Materials and methods}

\section{Preparation of antibiotic-loaded blocks}

HABs (Boneceram-P, Sumitomo Pharmaceutical Co. Ltd, Tokyo) with the following properties were used:

Received 19 Nov. 1996; revised version accepted 10 Feb. 1997. Corresponding author: Dr M. Itokazu. mol.wt, 1004.63; porosity, 50\%; pore size, 50 $300 \mu \mathrm{m}$; dimensions, $2 \times 2 \times 3 \mathrm{~mm}$. Fresh HABs were mixed with arbekacin (Meiji Seika Ltd, Tokyo, Japan) $400 \mathrm{mg}$ dissolved in $2 \mathrm{ml}$ of physiological saline solution, and centrifuged at $1500 \mathrm{rpm}$ for $15 \mathrm{~min}$. The antibiotic absorption rate by this method was $35 \% / 10$ $\mathrm{mm}^{3}$ volume, so that $0.84 \mathrm{mg}$ of arbekacin was captured into one experimental block.

\section{In-vivo study}

Wistar male rats weighing $200-250 \mathrm{~g}$, were used. The animals were anaesthetised with an intraperitoneal injection of pentobarbital sodium, $50 \mathrm{mg} / \mathrm{kg}$. The medullary cavity of the proximal tibia was opened and an 8-mm long silk thread soaked in a bacterial suspension containing $1.0 \times 10^{8}$ cfu of $S$. aureus IM242 (Institute of Anaerobic Bacteriology, Gifu University School of Medicine) was inserted. The cavity was then closed with bone wax under sterile conditions. The rats were fed a standard antibiotic-free rat diet throughout. The MIC of arbekacin for the $S$. aureus strain used was $0.25 \mu \mathrm{g} / 1$.

The HAB was implanted 4 weeks after the infection was induced. Each rat was re-anaesthetised and tibial $\mathrm{X}$-rays were obtained. All rats showed gross pathological and radiographical signs of osteomyelitis at this time (purulent material, widening of the proximal tibia and disruption or pitting of bone architecture). The right proximal tibia was disinfected, and then prepared 
for surgery. A $3 \times 5 \mathrm{~mm}$ rectangular window was cut through into the medullary cavity with a dental burr. At this point the rats were divided into four groups. The first group consisted of three animals that received no treatment for 4 weeks after the inoculation of $S$. aureus. In experimental group I $(\mathrm{n}=21)$ an arbekacin-loaded $\mathrm{HAB}$ was implanted into the marrow cavity of each rat after debridement and irrigation of the marrow cavity with $40 \mathrm{ml}$ of normal saline. In control group II $(n=21)$ rats were similarly given implants of drug-free HABs. Animals in group III $(\mathrm{n}=21)$ were treated with arbekacin $10 \mathrm{mg} / \mathrm{kg}$ daily for 1 week by subcutaneous injection after debridement and washing of the wound only.

\section{Bacteriological assessment}

The tibia was removed from soft tissues, crushed and homogenised with $2 \mathrm{ml}$ of saline under sterile conditions. Serial dilutions of this suspension were inoculated onto three plates of Mueller-Hinton Agar (Nissui Co. Ltd, Tokyo, Japan). After incubation for $48 \mathrm{~h}$, the viable count $(\mathrm{cfu} / \mathrm{ml})$ was calculated. Statistical significance was tested by Student's $t$ test.

\section{Radiological evaluation}

Radiographs of tibias were obtained with Softex film (Fuji Co. Ltd, Tokyo, Japan) 1 and 7 weeks after implantation of the HABs.

\section{Histological examination}

Two animals from each group were killed 1, 3, 5 and 7 weeks after the implantation. Each tibia was fixed with formalin $4 \%$, sliced into sections $3 \mu \mathrm{m}$ thick, and stained with haematoxylin and eosin. For the nondecalcified sections saw cuts of c. $500 \mu \mathrm{m}$ were made perpendicular to the long axis of the tibia. Tissues were milled down to $c .60 \mu \mathrm{m}$ with a micromilling device (Maruto Co. Ltd, Tokyo, Japan) and stained with toluidine blue.

\section{Pharmacokinetic studies}

Samples of serum were obtained from three rats $30 \mathrm{~min}$ after implantation of an arbekacin-loaded HAB into the marrow cavity of the tibia and at random times thereafter. The specimens of serum were kept at $-20^{\circ} \mathrm{C}$ until measurement of arbekacin concentrations by fluorescence polarisation immunoassay (Meijiseika Co. Ltd, Tokyo).

\section{Results}

\section{Bacteriological assessment}

Bacterial counts in untreated rats 4 weeks after infection with $S$. aureus averaged $2 \times 10^{6}$ SEM $2.7 \times 10^{6} \mathrm{cfu} / \mathrm{ml}$ of tibial homogenate. One week after implantation of arbekacin-loaded HABs bacterial counts averaged $1.8 \times 10^{4} \mathrm{SEM} 1.2 \times 10^{4} \mathrm{cfu} / \mathrm{ml}$; at 3 weeks average counts remained at $1.7 \times 10^{4} \mathrm{SEM}$ $0.9 \times 10^{4} \mathrm{cfu} / \mathrm{ml}$. After 5 and 7 weeks, bacteria were no longer detectable in some rats and were present in counts of $\left(1.5 \times 10^{3}\right)-\left(9.5 \times 10^{3}\right) \mathrm{cfu} / \mathrm{ml}$ in others (Table 1). In rats given drug-free implants, bacterial counts 1 week after the implantation were on average $1.6 \times 10^{5} \quad \mathrm{SEM} 0.4 \times 10^{5} \mathrm{cfu} / \mathrm{ml}$; counts remained above $3.8 \times 10^{6} \mathrm{SEM} 3.1 \times 10^{6} \mathrm{cfu} / \mathrm{ml}$ for the 7 -week period of observation. In rats treated with arbekacin by subcutaneous injection, bacterial counts after 1 week were $2.3 \times 10^{5} \mathrm{SEM} 1.8 \times 10^{5} \mathrm{cfu} / \mathrm{ml}$. Overall, total counts of bacteria in rats receiving arbekacin-loaded HAB implants $\left(1.5 \times 10^{4} \mathrm{SEM} 0.5 \times 10^{4} \mathrm{cfu} / \mathrm{ml}\right)$ were significantly lower than those observed in rats given drug-free implants $\left(2.1 \times 10^{5}\right.$ SEM $\left.1.0 \times 10^{5} \mathrm{cfu} / \mathrm{ml}\right)$ or drug injections only $\left(5.5 \times 10^{6} \mathrm{SEM} 4.6 \times 10^{6} \mathrm{cfu} /\right.$ $\mathrm{ml})(\mathrm{p}<0.01$ and $\mathrm{p}<0.05$, respectively). No bacterial growth (limit of detection $<1 \times 10^{2} \mathrm{cfu} / \mathrm{ml}$ ) was observed in five rats given the arbekacin-loaded implant, in one rat treated with antibiotic alone, and was not observed in any of the animals given a drugfree implant (Table 1).

\section{Radiological evaluation}

X-ray evaluation of the treatment with arbekacinloaded HAB showed that a radiolucent area surrounding the implant diminished gradually (Fig. 1). Almost all of the implants were united with bone and a radiolucent lesion with a periosteal reaction was observed in only one case. In rats given a drug-free implant, a radiolucent area persisted in the vicinity of the implant and osteosclerotic areas still existed after 7 weeks (Fig. 2). Osteolytic and extensive lesions were observed in all cases.

Table 1. Bacterial counts in tibial homogenates of rats experimentally infected with $S$. aureus

\begin{tabular}{lccc}
\hline \multirow{2}{*}{$\begin{array}{l}\text { Period after } \\
\text { treatment (weeks) }\end{array}$} & \multicolumn{3}{c}{ Viable count (cfu/ml of homogenate) in } \\
\cline { 2 - 4 } 1 & Group I & Group II & Group III \\
\hline $5.9 \times 10^{3}$ & $2.2 \times 10^{5}$ & $5.8 \times 10^{5}$ \\
& $4.2 \times 10^{4}$ & $1.0 \times 10^{5}$ & $3.1 \times 10^{4}$ \\
& $5.9 \times 10^{3}$ & $1.6 \times 10^{5}$ & $7.5 \times 10^{4}$ \\
3 & $4.0 \times 10^{3}$ & $1.3 \times 10^{6}$ & $4.5 \times 10^{6}$ \\
& $3.4 \times 10^{4}$ & $1.0 \times 10^{7}$ & $1.2 \times 10^{5}$ \\
& $1.4 \times 10^{4}$ & $8.0 \times 10^{4}$ & $5.6 \times 10^{7}$ \\
& & $2.3 \times 10^{6}$ & $4.3 \times 10^{6}$ \\
5 & $<1 \times 10^{2}$ & $6.3 \times 10^{5}$ & $3.3 \times 10^{3}$ \\
& $<1 \times 10^{2}$ & $1.2 \times 10^{6}$ & $1.4 \times 10^{3}$ \\
& $1.5 \times 10^{3}$ & $3.6 \times 10^{5}$ & $1.4 \times 10^{5}$ \\
7 & $9.5 \times 10^{3}$ & $1.3 \times 10^{5}$ & $5.0 \times 10^{2}$ \\
& $<1 \times 10^{2}$ & $1.4 \times 10^{6}$ & $<1 \times 10^{2}$ \\
& $<1 \times 10^{2}$ & $1.0 \times 10^{7}$ & $1.5 \times 10^{4}$ \\
& $<1 \times 10^{2}$ & & \\
\hline
\end{tabular}

Group I, rats with arbekacin-loaded HAB implant; group II, rats with antibiotic-free HAB implant; group III, rats treated with arbekacin alone (no implant). Difference between results in groups I and II statistically significant, $\mathrm{p}<0.01$. 

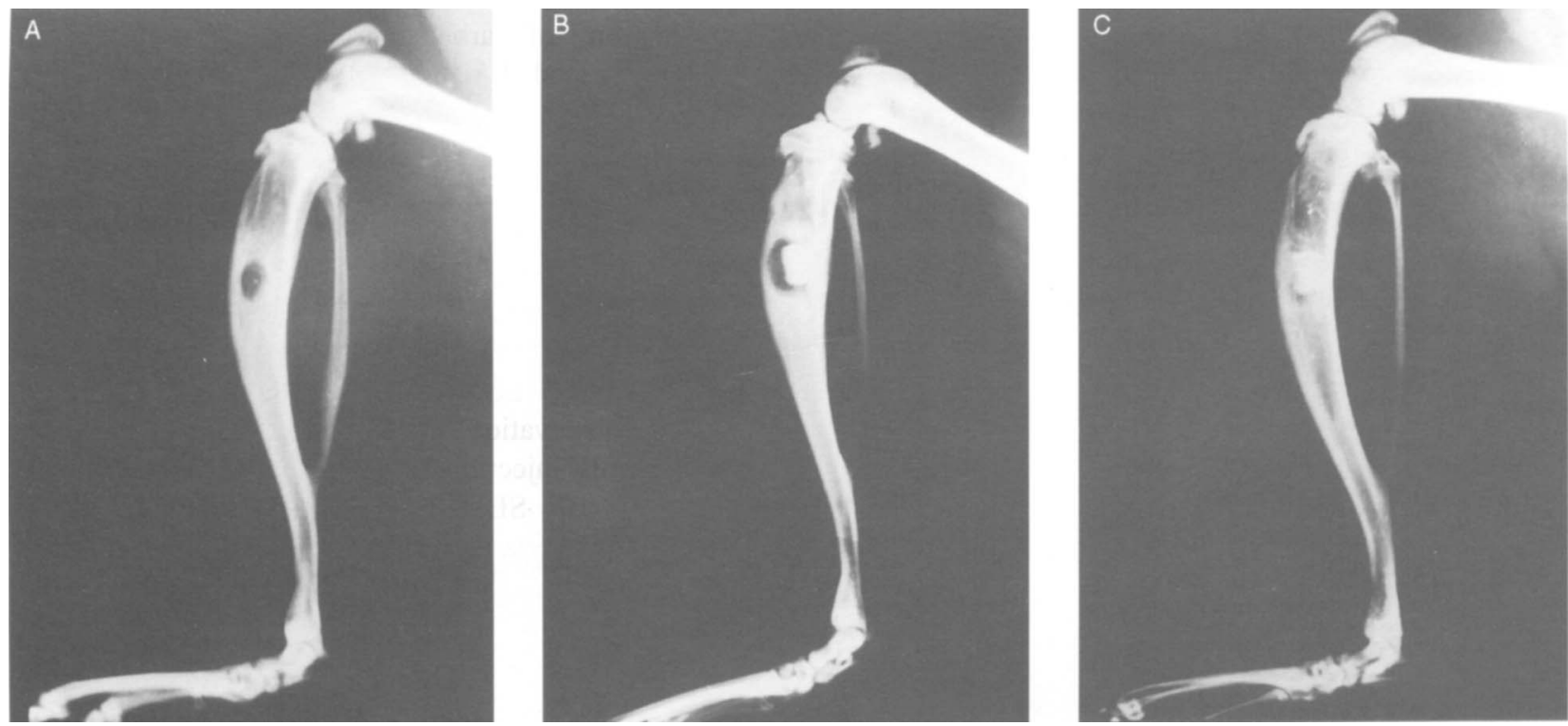

Fig. 1. X-rays of rat tibias: a, 1 week; b, 3 weeks; c, 7 weeks after implantation of an arbekacin-loaded HAB. After 3 weeks the radiolucent zone was diminished; after 7 weeks it had almost vanished.
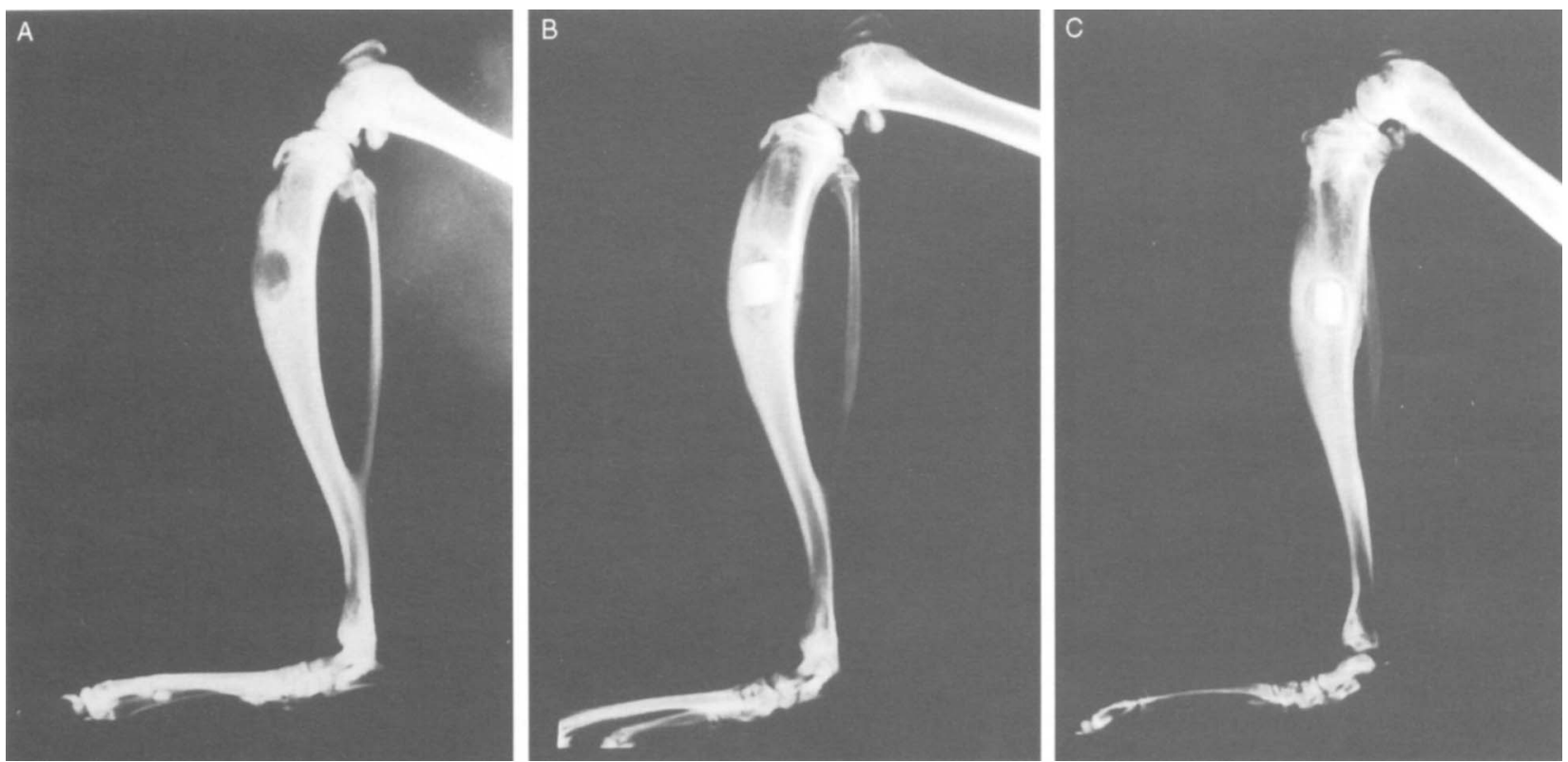

Fig. 2. X-rays of rat tibias: a, 1 week; b, 3 weeks; c, 7 weeks after implantation of an antibiotic-free HAB. After 3 weeks bone sclerosis surrounding the implant was visible; after 7 weeks osteosclerosis in the vicinity of the implant increased and a radiolucent zone clearly existed.

\section{Histological findings}

After implantation of arbekacin-loaded HABs, new bone formation was visible at the surface of the HAB and complete contact without fibrous tissue was evident at the interface between the bone and implant at 7 weeks (Fig. 3a,b). Few leucocytes were seen before 7 weeks without new bone formation. In animals given antibiotic-free HABs, leucocyte infiltration, proliferation of fibrous tissue and bone abscess formation were seen in the vicinity of the HAB (Fig. 3c).

\section{Pharmacokinetic studies}

The serum arbekacin concentration reached a peak of 8.7 SEM $1.4 \mu \mathrm{g} / \mathrm{ml}, 1 \mathrm{~h}$ after implantation (Fig. 4). Antibiotic was undetectable by immunoassay after $3 \mathrm{~h}$.

\section{Discussion}

Despite advances in antibiotic treatment, chronic osteomyelitis is still difficult to cure, as low concentra- 

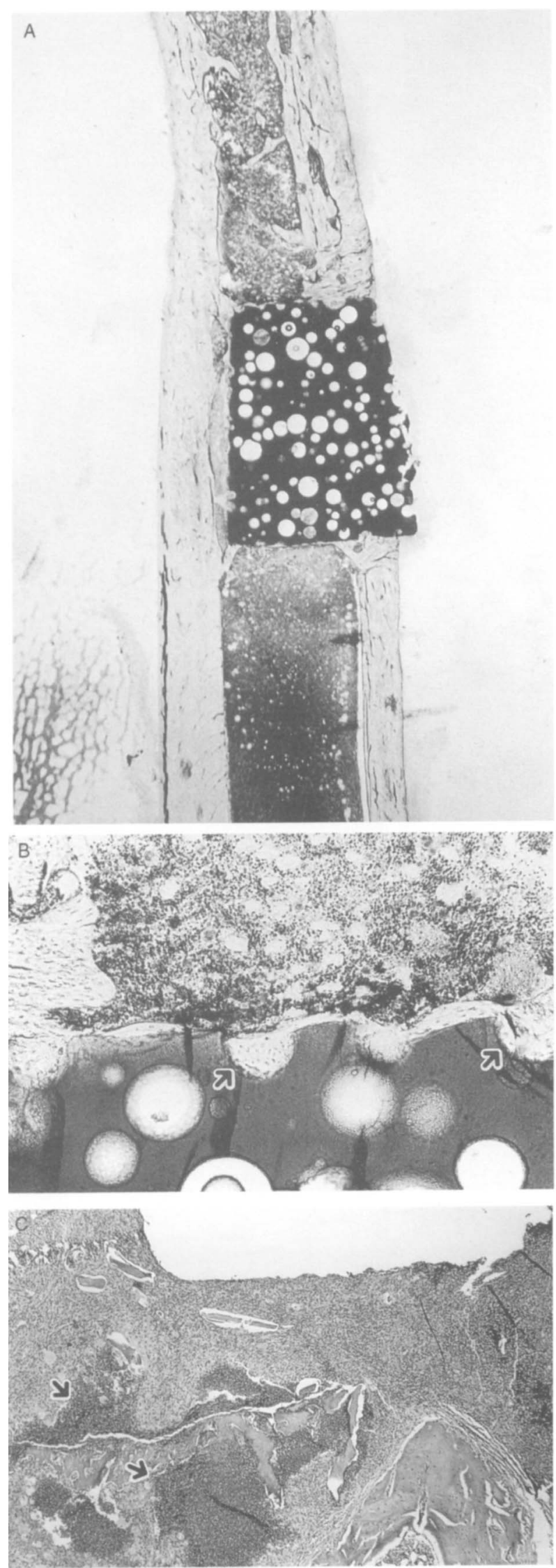

Fig. 3. Histopathological changes 7 weeks after implantation of HABs. Longitudinal section of bone with arbekacinloaded HAB: a, $\times 9 ; \mathbf{b}, \times 90$, showing block in contact with new bone (arrows) (non-decalcified toluidine blue staining); $\mathbf{c}$; section of bone with drug-free HAB $(\times 9)$ showing inflammatory cells and abscess formation (arrows).

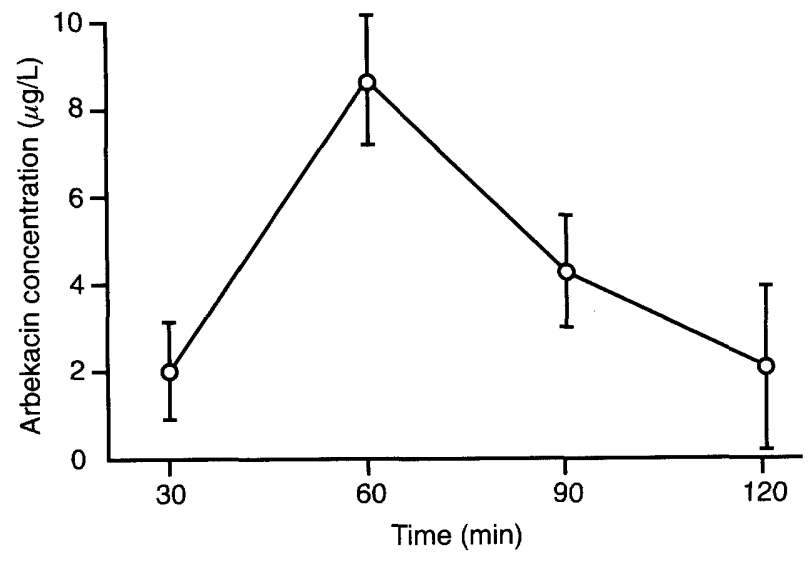

Fig. 4. Changes in serum arbekacin concentration after the implantation of antibiotic-loaded HAB $(0.84 \mathrm{mg}$ arbekacin/block).

tions of antibiotics are achieved in the lesion, because of bone necrosis, sclerosis and decreased blood flow. The causative bacteria are known to produce a large amount of extracellular glycocalyx material [2] and necrotic bone provides a surface suitable for the development of a biofilm [3]. For these reasons, the surgical removal of necrotic tissues, with long-term delivery of high concentrations of drug at the site of the lesion are the primary methods of treatment of chronic osteomyelitis.

Currently, antibiotic-loaded cement beads [4,5] of polymethylmethacrylate are successfully used as a drug delivery system. However, the local insertion of this compound has the disadvantage of requiring subsequent surgery for replacement with an autograft. Moreover, drugs that are resistant to the heat necessary for the production of polymethylmethacrylate must be chosen.

In the search for suitable carrier systems, various biological substances have been tested: tricalcium phosphate [6], plaster of Paris [7], a composite of D,L-lactic acid oligomer [8] and fibrinogen [9] have been investigated as absorbable carriers, mainly in experiments with animals. However, unlike hydroxyapatite, these materials would not be able to withstand mechanical forces. Hydroxyapatite has been widely used as a bioactive ceramic material for bone defects because of its excellent biocompatibility and nonantigenicity $[10,11]$. In a preliminary study, Shinto et al. [12] used porous calcium hydroxyapatite in which an antibiotic powder was packed into a cylindrical cavity. Yu et al. [13] also reported the experimental use of a self-setting hydroxyapatite cement formed into cement pellets.

Itokazu et al. recently described a drug-delivery system in which HABs were loaded with antibiotics by a centrifugation method [1]. The slow release of the activity of the aminoglycoside antibiotic arbekacin was demonstrated. A concentration of $0.5 \mu \mathrm{g} / \mathrm{L}$ 
(sufficient to inhibit most pathogens) was still maintained after 20 exchanges of phosphate-buffered saline over 40 days. In the present evaluation of the efficacy of arbekacin-loaded HABs in a rat model of osteomyelitis, bacterial counts in animals given implants of antibiotic-loaded blocks were lower than those in the control group throughout the experimental period. Moreover, complete cure was obtained in five of seven rats over a 5-week period of observation. Radiographical evaluation showed improvement in almost all animals with arbekacin-loaded implants. In contrast, deteriorating X-ray changes were seen in all animals given drug-free implants or subcutaneous arbekacin. Histological findings showed marked improvements in rats receiving antibiotic-loaded implants, confirming the benefit of the presence of antibiotics, although bacteria persisted in some lesions. Similar results have been reported in the treatment of experimental osteomyelitis of rabbits with gentamicinloaded plaster of Paris [7]. In that study bacteria persisted in most animals but there was marked clinical improvement.

Monitoring of serum arbekacin concentrations after implantation of the antibiotic-loaded HABs showed that a peak concentration was achieved after $1 \mathrm{~h}$. Antibiotic was undetectable $3 \mathrm{~h}$ after implantation. These results suggest that antibiotic-loaded HABs may be safe to use in combination with intravenous injections. Slow release of antibiotics from HABs produces high localised concentrations which cannot be attained by parenteral therapy because of the risk of side-effects. Antibiotic-loaded HABs prepared by centrifugation may offer a valuable new form of local antibiotic therapy primarily for strut graft and osteomyelitis. Such an approach may be particularly useful in treating bone and joints infected with methicillin-resistant $S$. aureus.

\section{References}

1. Itokazu M, Matsunaga T, Kumazawa S, Yang W. A novel drug delivery system for osteomyelitis using porous hydroxyapatite blocks loaded by centrifugation. J Appl Biomater 1995; 6: 167-169.

2. Mayberry-Carson KJ, Tober-Meyer B, Smith JK, Lambe DW, Costerton JW. Bacterial adherence and glycocalyx formation in osteomyelitis experimentally induced with Staphylococcus aureus. Infect Immun 1984; 43: 825-833.

3. Marrie TJ, Costerton JW. Mode of growth of bacterial pathogens in chronic polymicrobial human osteomyelitis. $J$ Clin Microbiol 1985; 22: 924-933.

4. van Foerster G, Buchholz HW, Lodenkamper H, Lodenkamper U. [Treatment of osteomyelitis with antibiotic cement.] Behandlung der Osteomyelitis mit Antibioticazement. Chirurg 1982; 53: 709-715.

5. Wahlig H, Dingeldein E, Bergmann R, Reuss $\mathrm{K}$. The release of gentamicin from polymethylmethacrylate beads. An experimental and pharmacokinetic study. $J$ Bone Joint Surg $[\mathrm{Br}]$ 1978; 60-B: $270-275$

6. Eitenmüller J, Schmidt KH, Peters G, Gellissen G, Weltin R, Reichmann W. Experimental and preliminary clinical experience with absorbable calcium phosphate granules containing an antibiotic or antiseptic for the local treatment of osteomyelitis. $J$ Hosp Infect 1985; 6 Suppl A: 177-184.

7. Dahners LE, Frunberburk CH. Gentamicin-loaded plaster of Paris as a treatment of experimental osteomyelitis in rabbits. Clin Orthop 1987; 219: 278-282.

8. Wei G, Kotoura Y, Oka M et al. A bioabsorbable delivery system for antibiotic treatment of osteomyelitis. The use of lactic acid oligomer as a carrier. J Bone Joint Surg [Br] 1991; 73-B: $246-252$.

9. Redle H, Schlag G, Stanek G, Hirschl A, Seelich T. In vitro properties of mixtures of fibrin seal and antibiotics. Biomaterials 1983; 4: 29-32.

10. Itokazu M, Matsunaga T. Arthroscopic restoration of depressed tibial plateau fractures using bone and hydroxyapatite grafts. Arthroscopy 1993; 9: 103-108.

11. Itokazu M, Matsunaga T, Ishii M, Kusakabe $H$. Use of arthroscopic and interporous hydroxyapatite as a bone graft substitute in tibial plateau fractures. Arch Orthop Trauma Surg 1996; 115: 45-48.

12. Shinto Y, Uchida A, Korkusuz F, Araki N, Ono K. Calcium hydroxyapatite ceramic used as a delivery system for antibiotics. J Bone Joint Surg [Br] 1992; 74-B: 600-604.

13. Yu D, Wong J, Matsuda Y, Fox LF, Higuchi Wl, Otsuka M. Self-setting hydroxyapatite cement: a novel skeletal drugdelivery system for antibiotics. J Pharm Sci 1992; 81: $529-531$. 